\title{
An Integrated Interactive Visualization and Analysis Environment to study the Impact of Fire on Building Structures
}

\author{
Dilip Banerjee* John Gross \\ Terence Griffin
}

\author{
Pradeep Reddy Gaddam \\ John Hagedorn
}

\author{
Marc Olano $^{\dagger}$ \\ John Kelso
}

\author{
William Hess \\ Steve Satterfield
}

Judith Terrill
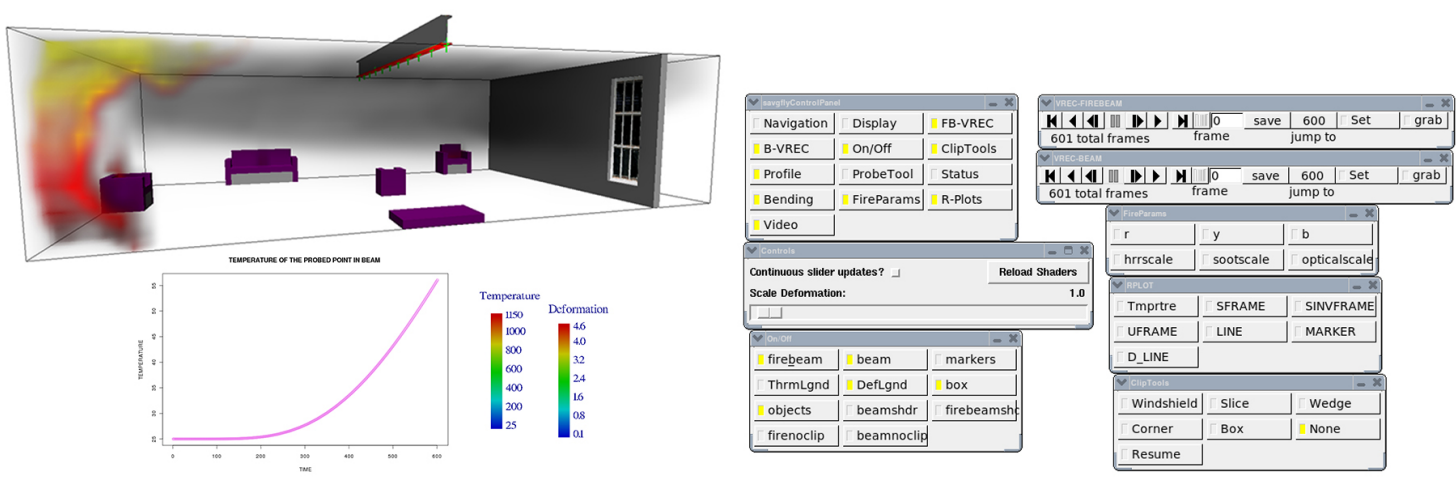

Figure 1: Our visualization and analysis environment. Upper left: $3 D$ visualization of fire, structure and room. Lower Left: example plot of temperature vs time for a user-selected point in the beam. Right: control panels for interacting with, and analyzing the visualization.

\section{Introduction}

In order to move away from the current prescriptive design methods towards performance based methods for the design of structures under fire, we need validated computer models. The next section describes our approach for modeling and analysis.

\section{Method}

We have created an integrated interactive visualization and analysis environment, that can be used immersively or on the desktop, to study the interaction among fire, heat transfer, and structural deformation computed from three linked simulations that model the fire, the heat transfer, and the structure.

In this initial study, we simulated the evolution of a fire in a room (with a small adjoining room) with furniture, a window and a single structural beam. A sequential process was followed in which first the NIST Fire Dynamics Simulator (FDS) [McGrattan 2007] was used to simulate the start and development of fire in the room. The adiabatic surface temperature at twelve marker points underneath the beams bottom flange location was used to capture the time dependent temperature during the FDS simulation. Then a second (finite element) computer program was used to calculate how the gas temperature at the markers propagated into the beam. Finally, a third (finite element) computer program was used to compute how the beam deformed over time due to combined effects of thermal and mechanical loads [Banerjee 2009]. The three programs produced three separate and very large datasets. The fire model was computed on 64,000 grid points for 115,705 time steps, and 6 scalars were studied for each of these points. The heat transfer and the structure models were computed on 10,332 elements and 12,642 nodes for each time step and two scalars, two vectors and two tensors were computed in total.

We used polygonal visualizations for the room and its contents, including the beam, and shaded the beam according to its tempera-

\footnotetext{
*Except as indicated, all authors are from NIST

${ }^{\dagger}$ Univ. of Maryland, Baltimore County
}

ture distribution. We used a ray traced volume shader to visualize the fire and smoke. We combined temperature, heat release rate per unit volume, and soot density to come up with a local color and opacity at each step along the ray. Many volume renderers assume that the material has a constant color and opacity within any given step of the numeric integration. Instead, we used an approximation of linear attenuation within each step [Moreland 2004]. We used the open dynamics physics engine [ODE ] to display window breakage at a time indicated in the fire simulation. To assist in the analysis, we can interactively display numeric values at individual points in the $3 \mathrm{D}$ space, and we can plot time histories of: temperature, the stress tensor in the beam, and deformation of the beam (location and orientation). We can also plot output values along a chosen line.

\section{Conclusion and Future Work}

Preliminary investigation using our environment, provided initial validation of the linked model approach. The visualization and analysis environment was crucial to develop an understanding of the output of the three simulations. But this was a very simple case developed to validate the methodology. Future work consists of further validation, and more complex building and fire scenarios. Additionally, we will be studying how to best perform the analysis in the context of the visualization in order to advance the modeling effort and our understanding.

\section{References}

BANERJEE, D. 2009. Visualization of structural behavior under fire. Technical Report NISTIR 7619, NIST, August.

MCGRATtAN, K. B. 2007. Fire dynamics simulator (version 5). Technical Report NIST SP 1018-5, NIST, October.

Moreland, K. 2004. Fast High Accuracy Volume Rendering. $\mathrm{PhD}$ thesis, University of New Mexico.

Open Dynamics Engine [online]. http://www.ode.org/. 